\title{
Der einsame Läufer
}

\author{
Matthias Schymura und Jörg M. Wills
}

\begin{abstract}
Im November 2017 erschien in der großen holländischen Tageszeitung NRC Handelsblad ein doppelseitiger bebilderter Artikel mit dem Titel „De eenzame loper“ [12]. Das ist viel Platz für ein einzelnes mathematisches Problem, das zudem bisher nur wenige Anwendungen innerhalb der Mathematik hat.

Das Problem - die „Lonely Runner Conjecture“ - hat jedoch zwei wesentliche Eigenschaften für einen populärwissenschaftlichen Artikel: Es ist so einfach zu formulieren, dass es auch ein mathematischer Laie versteht; und es ist noch ungelöst, obwohl sich schon etwa 40 Mathematiker und Mathematikerinnen, darunter der Fields-Medaillen-Träger Terry Tao, in über zo Publikationen daran die Zähne ausgebissen haben.
\end{abstract}

\begin{abstract}
Das Einsame-Läufer-Problem. Auf einer Kreisbahn der Länge 1 drehen $n$ Läufer ihre Runden. Sie sind gleichzeitig vom selben Ort aus gestartet und laufen mit konstanter, paarweise verschiedener Geschwindigkeit. Ein Läufer heißt einsam, wenn er oder sie von allen anderen Läufern einen Abstand von mindestens $\frac{1}{n}$ hat.
\end{abstract}

Vermutung: Jeder Läufer ist irgendwann mal einsam.

Für zwei Läufer ist das Problem sofort gelöst: Irgendwann befinden sie sich auf gegenüberliegenden Bahnpunkten. Ab drei Läufern wird es schrittweise schwieriger. Trotzdem ist es kaum zu glauben, dass die Vermutung bisher nur für bis zu sieben Läufer bestätigt ist.

Abbildung 1 zeigt das Problem für vier Läufer. Im Internet, z. B. in der Wikipedia [3], gibt es animierte Illustrationen mit mehreren Läufern in Aktion. Die obige poetische Formulierung stammt von dem kanadischen Mathematiker Luis Goddyn, der sie 1998 kreierte. Sie hat sich als populärste Version durchgesetzt und nicht zuletzt auch zu neuen Varianten angeregt.
Das Problem des einsamen Läufers ist aber ganze 33 Jahre älter und trug vorher auch andere, etwas weniger attraktive Namen. Es entstand 1965 als Gegenstück zu Hermann Weyls Gleichverteilungssatz. Dieser besagt, dass die ganzzahligen Vielfachen eines Vektors von $n$ Irrationalzahlen im Einheitswürfel mod 1 gleichverteilt sind. Dies ist ein Maß für die besonders gute simultane Approximation von Irrationalzahlen durch rationale Zahlen - ein uraltes mathematisches Problem.

Weyls Satz gilt aber nur, wenn die Irrationalzahlen untereinander nicht rational abhängig sind, wie man seit Kronecker (1885) und eben Weyl (1916) weiß. Im Fall, dass rationale Abhängigkeiten existieren, liegen die Vektoren auf linearen Unterräumen mod 1 und die Approximation ist schlecht. Sie ist besonders schlecht, wenn die rationale Abhängigkeit maximal ist. Denn dann sind die Vektorkomponenten rationale (bzw. ganzzahlige) Vielfache einer einzigen Irrationalzahl, und die Vielfachen des Vektors liegen alle auf einer Geraden mod 1. Es bleibt also nur noch die Frage nach den $n$ ganzen Zahlen, für die die Approximation am schlechtesten ist. Damit war das Problem geboren.

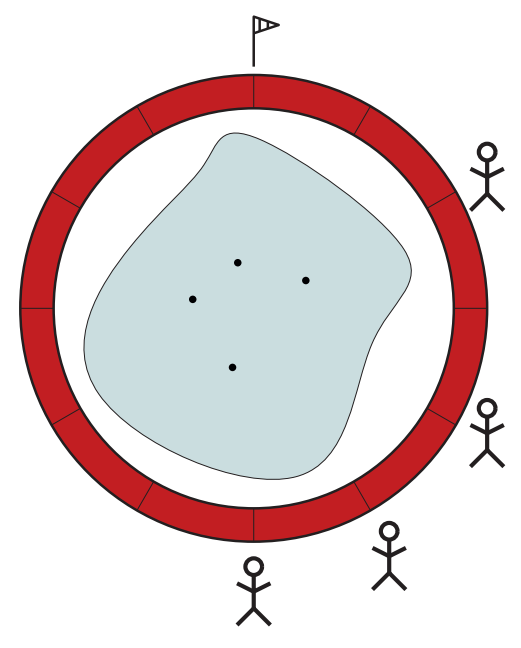

$t=\frac{1}{12}:$ Niemand ist einsam.

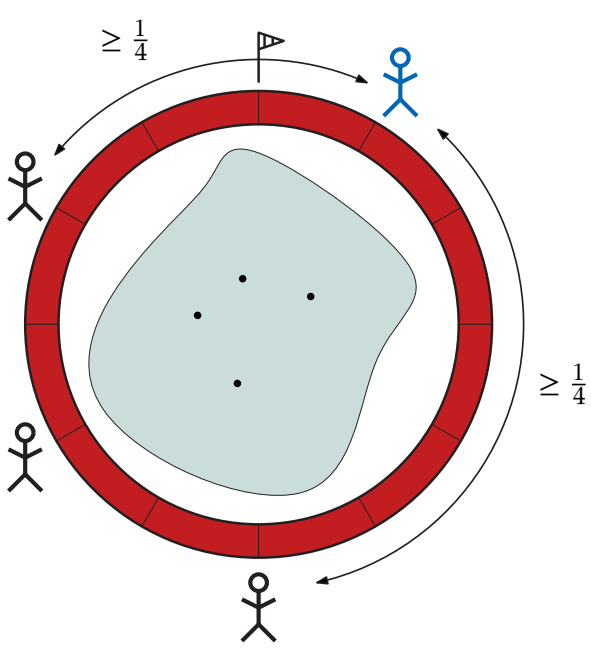

$t=\frac{5}{12}$ : Der blaue Läufer ist einsam.

Abbildung 1. Vier Läufer mit den Geschwindigkeiten 2, 4, 5 und 6 zu verschiedenen Zeitpunkten $t$ 
In drei kleinen Arbeiten von 1965, 1967 und 1968 hat Wills $[13,14]$ die Fragestellung an einem Anwendungsbeispiel skizziert, eine erste einfache Schranke bewiesen und eine optimale Schranke inklusive der kanonischen Gleichheitsfälle vermutet.

Obwohl das Problem aus der schlechten Approximation von Irrationalzahlen entstand, spielen die Irrationalzahlen hier gar keine Rolle, sondern nur die rationalen Abhängigkeiten zwischen ihnen. Es ist also eine Fragestellung in der Geometrie der Zahlen.

\section{Sichtbarkeiten und Billards}

Das Problem des einsamen Läufers wurde über die Jahre hinweg auf verschiedenste Weise neu, aber mathematisch äquivalent, interpretiert. Jede dieser Versionen hat ihren eigenen Charme und erlaubt die Benutzung von Methoden aus verschiedenen Teildisziplinen der Mathematik. Die folgende Beobachtung liegt all diesen Interpretationen $\mathrm{zu}$ Grunde: Die relativen Abstände der Läufer zu einem beliebigen Zeitpunkt hängen nur von den relativen Geschwindigkeiten der Läufer untereinander $a b$.

Mit anderen Worten, es ist irrelevant ob alle Läufer um den selben Geschwindigkeitsbetrag simultan schneller oder simultan langsamer laufen. Als Konsequenz erhalten wir, dass das Einsame-Läufer-Problem äquivalent zum „FaulenLäufer-Problem “ ist, bei dem einer der Läufer am Startpunkt stehen bleibt und wir uns nur für dessen Einsamkeit interessieren.

Schreiben wir nun $v_{i}$ für die Geschwindigkeit des $i$ ten (fleißigen) Läufers, so sehen wir, dass eine Instanz des Faule-Läufer-Problems mit $n$ Läufern zu einem Vektor $v=\left(v_{1}, \ldots, v_{n-1}\right)$ im $\mathbb{R}^{n-1}$ korrespondiert, den wir im folgenden als Geschwindigkeitsvektor bezeichnen. Diese geometrische Deutung hat Cusick [4] genutzt und 1973 die folgende ebenso elegante wie anschauliche Version formuliert:

Das Sichtbarkeitsproblem. Das Standardgitter $\mathbb{Z}^{n-1}$ induziert eine Zerlegung des $\mathbb{R}^{n-1}$ in Einheitswürfel. In jedem dieser Einheitswürfel liegt ein kleinerer Würfel, dessen Seitenflächen von den entsprechenden Seitenflächen des Einheitswürfels den Abstand $\frac{1}{n}$ haben.

Vermutung: Jeder vom Koordinatenursprung ausgehende Lichtstrahl, der nicht parallel zu einer der Würfelseiten verläuft, wird durch einen der kleinen Würfel blockiert.

Die Lichtstrahlen gehen dabei in die durch den Geschwindigkeitsvektor $v$ bestimmte Richtung vom Ursprung aus. Abbildung 2 zeigt Cusicks Version in der Ebene, das heißt für $n=3$ Läufer. Zur Illustration haben wir den Lichtstrahlen verschiedene Farben gegeben. Der gelbe Strahl mit Richtungsvektor $v=(2,1)$ zeigt, dass der Abstand $\frac{1}{3}$ maximal ist, denn er wird an der unteren rechten Ecke des ersten Würfels blockiert. Wenn wir ihn uns weiterdenken (gestrichelte Linie), dann wird er andere Würfel auch höchstens an deren Ecken treffen. In beliebiger Dimension zeigt der Geschwindigkeitsvektor $v=(1,2, \ldots, n-1)$, dass der Abstand $\frac{1}{n}$ maximal ist.

Drei Jahre nach Cusick formulierte Isaac Schoenberg [10] eine gleichwertige und ebenso elegante Version:

Das Billard-Problem. Wir spielen Billard auf einem ( $n-$ 1)-dimensionalen würfelförmigen Billardtisch der Kantenlänge 1. In der Mitte des Tisches ist ein würfelförmiges Hindernis platziert, dessen Seitenflächen von den entsprechenden Tischseiten den Abstand $\frac{1}{n}$ haben.

Vermutung: Bei jedem Billardstoß, der von einer Ecke ausgeht und ins Tischinnere verläuft, wird die Kugel das Hindernis treffen.

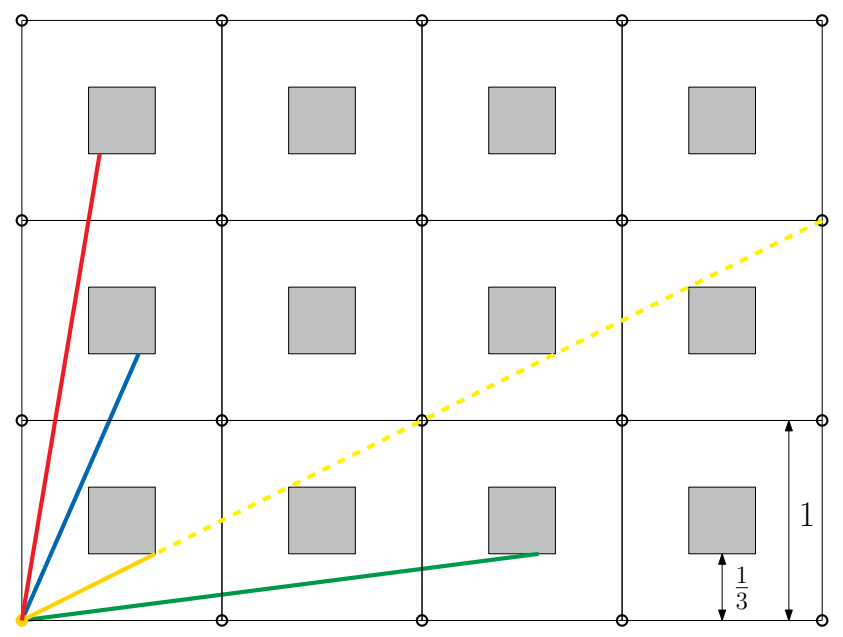

Abbildung 2. Das Sichtbarkeitsproblem im $\mathbb{R}^{2}$ 


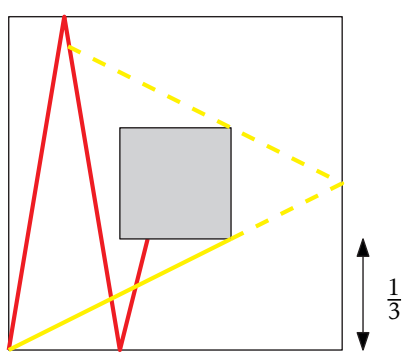

(a)

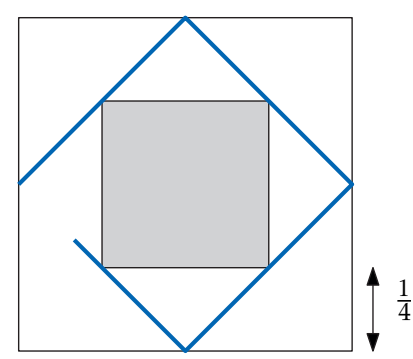

(b)

Abbildung 3. Das Billard-Problem im $\mathbb{R}^{2}$

Der gelbe bzw. rote Billardstoß in Abbildung 3 (a) korrespondiert zum gelben bzw. roten Lichtstrahl im Sichtbarkeitsproblem in Abbildung 2.

Am Billard-Problem lässt sich sehr gut erkennen, dass es ausschlaggebend ist, die Kugel von einer Ecke aus zu stoBen. Erlaubt man einen beliebigen Ausgangspunkt für den Stoß, so müssen wir das Hindernis natürlich etwas größer machen um alle Kugelbahnen zu behindern. Ein solches Beispiel ist die blaue Billardbahn in Abbildung 3 (b), die ein Hindernis der Kantenlänge $\frac{1}{2}$ erfordert. Schoenberg [10] löste dieses relaxierte Billard-Problem vollständig und zeigte, dass das Hindernis auf dem $(n-1)$-dimensionalen Billardtisch eine Kantenlänge von $\frac{n-2}{n-1}$ haben muss, und dass dies bestmöglich ist.

Neben den Interpretationen von Cusick, Schoenberg und Wills gibt es viele weitere Querverbindungen innerhalb der Mathematik, von denen wir einige hier kurz erwähnen möchten.

- Eine weitere geometrische Darstellung wurde von Malikiosis \& Schymura [8] gefunden. Die Aufgabe besteht darin, einen ganzzahligen Punkt in einer Projektion des Würfels (Zonotop) zu finden, die durch die Geschwindigkeiten der Läufer definiert ist.

- Zhu [15, Abschnitt 7] hat erkannt, dass das EinsameLäufer-Problem äquivalent zu einem speziellen Färbungsproblem auf sogenannten Distanzgraphen ist. Damit erhielt die Fragestellung Zugang zur Graphentheorie und weckte das Interesse eines noch viel breiteren mathematischen Publikums.

- Bienia et al. [2] diskutieren eine graphentheoretische Anwendung des einsamen Läufers auf nirgends verschwindende Flüsse in Graphen.

Diese Fülle von Interpretationen hat zu starken Teilresultaten geführt, auf die wir weiter unten eingehen. Die vollständige Lösung des Problems liegt aber noch in weiter Ferne und man fragt sich zu Recht:

\section{Warum ist das Problem so schwierig?}

\section{Indiz}

Die Komplexität der bis jetzt bekannten Beweise für kleine Anzahlen von Läufern steigt rasch an.

Für zwei Läufer ist das Problem wie oben gesehen klar, für drei Läufer einfach, aber $\mathrm{ab} n=4$ wird es bereits schwie- rig. Dieser Fall wurde unabhängig von Betke \& Wills (1972) und Cusick (1973) behandelt; $n=5$ geht auf Cusick \& Pomerance (1984) und Bienia et al. (1998) zurück; $n=6$ konnten Bohman, Holzman \& Kleitman (2001) erledigen. Renault [9] hat 2004 eine einheitliche und prägnante Argumentation für $n \leq 6$ gefunden, die aber für 7 Läufer wiederum fehl schlägt. Barajas \& Serra [1] landeten den bisher letzten Coup und bewiesen die Vermutung für $n=7$. Sobald mindestens 8 Läufer auf der Bahn sind, gibt es nur noch Teilbeweise unter starken Einschränkungen an die Geschwindigkeiten.

2. Indiz

Die Extremalfälle scheinen sehr unstrukturiert zu sein.

Wie bereits oben angedeutet, reicht es aus, Läufer mit ausschließlich ganzzahligen Geschwindigkeiten zuzulassen. Sobald es rationale Unabhängigkeiten unter den Geschwindigkeiten gibt, wird das Problem einfach. Dies zeigt bereits, dass der einsame Läufer für die meisten Geschwindigkeitsvektoren gelöst ist. Ähnliche Resultate sind in den Arbeiten von Tao [11] und Czerwiński [5] enthalten.

Unter den verbleibenden Fällen sind die kanonischen extremalen Geschwindigkeitsvektoren $(1,2, \ldots, n-1)$ bei weitem nicht die einzigen. Peter Flor gab 1968 (siehe [14]) die ersten beiden „Ausreißer“ an: Für fünf fleißige Läufer mit Geschwindigkeiten 1, 3, 4, 5, 9 und für sieben fleißige Läufer mit Geschwindigkeiten 1, 2, 3, 4, 5, 7, 12 ist der maximale Abstand $\frac{1}{6}$ bzw. $\frac{1}{8}$. Goddyn \& Wong [7] haben mit Computerhilfe folgende Tabelle erstellt, die alle nicht-kanonischen Extremalfälle für höchstens 20 fleißige Läufer mit Höchstgeschwindigkeit 40 auflistet.

\begin{aligned} & \hline$n$ Extremale Geschwindigkeitsvektoren \\ & \hline 5$(1,3,4,7) \\ & 6(1,3,4,5,9) \\ & 8(1,4,5,6,7,11,13) \\ & 8(1,2,3,4,5,7,12) \\ & 14(1,2,3,4,5,6,7,8,9,10,11,13,24) \\ & 20(1,2,3,4,5,6,7,8,9,10,11,12,13,14,15,16,17,19,36) \\ &$\hline\end{aligned}

\section{Indiz}

Die allgemeine Schranke an den maximalen Abstand $\lambda$, den alle fleißigen Läufer vom faulen Läufer gleichzeitig erreichen können, wurde innerhalb von 50 Jahren trotz großer Anstrengung nur geringfügig verbessert. 
Nach Wills' Schranke

$$
\lambda \geq \frac{1}{2(n-1)}
$$

im Jahr 1968 (siehe [14]) hat es vier Arbeiten gegeben, die

$$
\lambda \geq \frac{1}{2(n-1)}+\frac{c}{n^{2}}
$$

für schrittweise größer werdendes $c>0$ garantieren. Das beste derzeitige Ergebnis ist von Terry Tao [11], der folgende Schranke beweist:

$$
\lambda \geq \frac{1}{2(n-1)}+\frac{c \log n}{n^{2}(\log \log n)^{2}}, \quad \text { für ein } c>0 .
$$

Die Form dieses Ausdrucks lässt den erforderlichen Aufwand erahnen, und er ist doch noch recht weit von dem Ziel $\lambda=\frac{1}{n}$ entfernt. Vielleicht wichtiger als die bewiesene Schranke: Tao skizziert, warum man mit den bisherigen Methoden nicht mehr als

$$
\lambda \geq \frac{1}{2(n-1)}+\frac{c \log n}{n^{2}}
$$

wird beweisen können. Das heißt: Für die Lösung des Einsame-Läufer-Problems brauchen wir neue Ideen und frische Ansätze.

\section{Wie geht es weiter?}

Es wird weitere Verbesserungen der Schranke an $\lambda$ geben, es wird vielleicht der Fall von acht Läufern geklärt, und man wird weitere interessante Varianten, wie zuletzt die der unsichtbaren Läufer von Czerwiński \& Grytczuk [6] ersinnen.

Trotz dieser großen Aufmerksamkeit, die die Vermutung besonders in den letzten Jahren bekommt, ist die „Lonely Runner Conjecture“ weiterhin offen. Es könnte sich auch einfach herausstellen, dass die Vermutung schlichtweg falsch ist und es zum Beispiel bereits für $n=8$ oder gar erst für $n=54239$ Läufer einen Geschwindigkeitsvektor gibt, für den die Schranke $\frac{1}{n}$ nie von allen fleißigen Läufern gleichzeitig erreicht wird.
Wahrscheinlich wird die Frage eines Tages vollständig gelöst werden, positiv oder negativ. Aber bis dahin müssen die Läufer wohl noch einige Runden drehen.

\section{Literatur}

[1] Javier Barajas and Oriol Serra. The lonely runner with seven runners. Electron. J. Combin., 15(1):Research paper 48, 18 pp. (electronic), 2008.

[2] Wojciech Bienia, Luis Goddyn, Pavol Gvozdjak, András Sebő, and Michael Tarsi. Flows, View Obstructions, and the Lonely Runner. J. Combin. Theory Ser. B, 72(1):1-9, 1998.

[3] Wikipedia contributors. Lonely runner conjecture - Wikipedia, The Free Encyclopedia. https://en.wikipedia.org/wiki/Lonely_ runner_conjecture, 2017.

[4] Thomas W. Cusick. View-obstruction problems. Aequat. Math., 9:165-170, 1973 .

[5] Sebastian Czerwiński. Random runners are very lonely. J. Combin. Theory Ser. A, 119(6):1194-1199, 2012.

[6] Sebastian Czerwiński and Jarosław Grytczuk. Invisible runners in finite fields. Inform. Process. Lett., 108(2):64-67, 2008.

[7] Luis Goddyn and Erick B. Wong. Tight instances of the lonely runner. Integers, 6:\#A 38, 14 pp., 2006.

[8] Matthias Henze and Romanos-Diogenes Malikiosis. On the covering radius of lattice zonotopes and its relation to viewobstructions and the lonely runner conjecture. Aequat. Math., 91(2):331-352, 2017 .

[9] Jérôme Renault. View-obstruction: A shorter proof for 6 lonely runners. Discrete Math., 287(1):93-101, 2004.

[10] Isaac J. Schoenberg. Extremum problems for the motions of a billiard ball. II. The $L_{\infty}$ norm. Nederl. Akad. Wetensch. Proc. Ser. A 79=Indag. Math., 38(3):263-279, 1976.

[11] Terence Tao. Some remarks on the lonely runner conjecture. Contrib. Discrete Math. (to appear), https://arxiv.org/abs/1701. O2048, 2017.

[12] Alex van den Brandhof. De eenzame loper. NRC Handelsblad, 2017. https://www.nrc.nl/nieuws/2017/11/24/ de-eenzame-loper-14211777-a1582547.

[13] Jörg M. Wills. Zwei Sätze über inhomogene diophantische Approximation von Irrationalzahlen. Monatsh. Math., 71:263-269, 1967.

[14] Jörg M. Wills. Zur simultanen homogenen diophantischen Approximation. I. Monatsh. Math., 72:254-263, 1968.

[15] Xuding Zhu. Circular chromatic number: a survey. Discrete Math., 229(1-3):371-410, 2001.

\footnotetext{
Dr. Matthias Schymura, École Polytechnique Fédérale de Lausanne, Institute for Mathematics, MA C1 563, Station 8, 1015 Lausanne, Schweiz matthias.schymura@epfl.ch

Prof. Dr. Dr.h.c. Jörg M. Wills, Universität Siegen, Emmy-Noether-Campus, Mathematisches Institut, Walter-Flex-Straße 3, 57068 Siegen wills@mathematik.uni-siegen.de
}

\footnotetext{
Matthias Schymura ist Postdoktorand in der Gruppe „Diskrete Optimierung“ an der EPFL in Lausanne, Schweiz. Zuvor war er im Rahmen der Exzellenzinitiative an der FU Berlin in der Arbeitsgruppe „Diskrete Geometrie" tätig und promovierte 2012 an der Otto-von-Guericke-Universität in Magdeburg.

Jörg M. Wills hat 1965 an der TU Berlin promoviert. Arbeitsgebiete: Diskrete Geometrie, insbesondere Geometrie der Zahlen, Finite Kugelpackungen, Polyeder, Konvexgeom. Seit 1974 Professor an der Universität Siegen. Drei Bücher (mit Peter M. Gruber). Forschungsaufenthalte: Beijing (Academia Sinica), Mexico City, Toronto, Calgary/Banff, Budapest (Acad.), Wien (Schrödinger), Universität Turin, Universität Triest. 1994-2004 Minerva (deutsch-israel. Wiss. Koop/Max-Planck). 2002 emeritiert, 2003 Dr. h. c. TU Wien.
} 